\title{
Erratum
}

\section{Erratum: Hu et al., "Conserved Tao Kinase Activity Regulates Dendritic Arborization, Cytoskeletal Dynamics, and Sensory Function in Drosophila"}

In the article, "Conserved Tao Kinase Activity Regulates Dendritic Arborization, Cytoskeletal Dynamics, and Sensory Function in Drosophila," by Chun Hu, Alexandros K. Kanellopoulos, Melanie Richter, Meike Petersen, Anja Konietzny, Federico M. Tenedini, Nina Hoyer, Lin Cheng, Carole L.C. Poon, Kieran F. Harvey, Sabine Windhorst, Jay Z. Parrish, Marina Mikhaylova, Claudia Bagni, Froylan Calderon de Anda, and Peter Soba, which appeared on pages 1819-1833 of the February 26, 2020 issue, the authors would like to correct the grant numbers.

The corrected grant numbers are as follows: M.R. and F.C.d.A. were supported by Deutsche Forschungsgemeinschaft FOR 2419, CA1495/1-1, and CA 1495/4-1 and JPND (Bundesministerium für Bildung und Forschung, 01ED1806). F.C.d.A. and P.S. were supported by Landesforschungsförderung Hamburg LFF-FV27 and ERA-NET NEURON (Bundesministerium für Bildung und Forschung, 01EW1910). This has been updated online.

DOI: 10.1523/JNEUROSCI.1334-20.2020 